\title{
Physico-Chemical and Heavy Metals in the Groundwater Samples Collected from Arsenic Endemic Areas of Shuklaganj (Unnao)
}

\author{
Anju Agrawal", Nis hi Kumar Shukla \\ Department of Zoology, S N Sen B V P G College, CSJM University, Kanpur, India
}

\begin{abstract}
Human health is greatly affected by exposure to arsenic through drinking water. Arsenic is a carcinogen and its consumption can negatively affect the gastrointestinal tract, cardio-vascular and central nervous systems. World Health Organisation (WHO) and US Environ mental Protection Agency have set the maximu m acceptable levelof arsenic in drin king water as $10 \mu \mathrm{g} / \mathrm{L}$. Attempts have been made to determine and establish a database on the drinking water quality of Shuklaganj area with particular emphasis on the physico-chemical characteristics and levels of heavy metals in the water samples. The physico-chemical parameters determined were $\mathrm{pH}$, hardness, alka lin ity, conductivity, TDS, salinity and ch loride content. The samples were collected in premonsoon and postmonsoon of Shuklaganj area. The $\mathrm{pH}$ of all the samples varied from 7.0 to 8.5 in both premonsoon and postmonsoon period. Hardness during in premonsoon varied from $180-212 \mathrm{mg} / \mathrm{L}$ and in postmonsoon varied fro $\mathrm{m} 140-210 \mathrm{mg} / \mathrm{l}$. Alkalinity varied fro $\mathrm{m} 84-112 \mathrm{mg} / \mathrm{L}$ in premonsoon and $60-128 \mathrm{mg} / \mathrm{L}$ in postmosoon. However conductivity was quite high and it ranged from $320-2140 \mu \mathrm{s} / \mathrm{cm}$ in premonsoon and $358-1944 \mu \mathrm{s} / \mathrm{cm}$ during postmonsoon. The TDS ranged from 181-1019 mg/l in premonsoon samples and $163-1102 \mathrm{mg} / 1$ in postmonsoon. Salinity varied from 0.0-1.0 ppt in premonsoon and 0.0-1.3 ppt in postmonsoon. Chloride content varied from to 12.1-36.4 in premonsoon and 8.08-44.5 $\mathrm{mg} / \mathrm{L}$ in potmonsoon. The picture of heavy metals and arsenic present in the water collected during premonsoon and postmonsoon periods were also determined. However, in premonsoon samples $\mathrm{Cr}, \mathrm{Cd}$ and $\mathrm{Ni}$ were absent in most of the samples. It was noticed that $\mathrm{Cd}$ and $\mathrm{Ni}$ content was almost absent in the samples collected during postmonsoon season. Copper varied from 0.0 to $0.0178 \mathrm{mg} / \mathrm{L}$ during premonsoon and 0.0002 to $0.0098 \mathrm{mg} / \mathrm{L}$ during postmonsoon. Zinc content varied from 0.0 to 3.26 in premonsoon period and fro $\mathrm{m} 0.0-3.6 \mathrm{mg} / \mathrm{L}$ in postmonsoon period. Iron varied from 0.0 to $17.99 \mathrm{mg} / \mathrm{L}$ in pre monsoon and varied from 0.0692 to 12.53 in postmonsoon samples. Manganese varied from 0.0 to $0.4454 \mathrm{mg} / \mathrm{L}$ in premonsoon samples and $0.0018-4.74 \mathrm{mg} / \mathrm{L}$ in premonsoon samples. Arsenic in premonsoon season varied from $0-250 \mathrm{ppb}$ and from $0-250 \mathrm{ppb}$ in postmonsoon. It is seen that with increase in $\mathrm{pH}$ above 8.5 , Arsenic desorbs from the oxide surfaces, thereby increasing concentration of Arsenic in solution. It is suggested that the most desirable and significant mechanism for the groundwater Arsenic problems is due to oxidising conditions and desorption of Arsenic from Arsenic contaminated sediments at high $\mathrm{pH}$.
\end{abstract}

Keywords Arsenic, Heavy Metals, Handpumps

\section{Introduction}

Human health is greatly affected by exposure to arsenic through drinking water. Arsen ic is a carcinogen and its consumption can negatively affect the gastrointestinal tract, cardio-vascular and central nervous systems. World Health Organ isation (WHO) and US Environmental Protection Agency have set the maximum acceptable level of arsenic in drinking water as $10 \mu \mathrm{g} / \mathrm{L}[1,2]$. Arsenic occurs in groundwater primarily as a result of natural weathering of

* Corresponding author:

anjuagrawal2@gmail.com (Anju Agrawal)

Published online at http://journal.sapub.org/als

Copyright (C) 2012 Scientific \& Academic Publishing. All Rights Reserved arsenic containing rocks, although in certain areas high arsenic concentration are caused due to industrial waste discharges and application of arsenical herbicides/pesticides [3]. Arsenic is present in water mainly in the forms of arsenate As (V) and arsenite (As III). In the environmentally relevant $\mathrm{pH}$ range 4-10, the dominant $\mathrm{As}(\mathrm{V})$ species are negatively charged $\left(\mathrm{H}_{2} \mathrm{AsO}_{4}{ }^{2-}\right)$, while the dominant As (III) species is neutrally charged $\left(\mathrm{H}_{3} \mathrm{AsO}_{3}\right)$.

Arsenic is introduced in the soil and groundwater during weathering of rocks and minerals followed by leaching and runoff. Also it can be introduced in soil and groundwater from anthropogenic sources. Many factors control Arsenic concentration and transport in groundwater which include Redox potential, absorption/desorption/precipitation/dissolu tion. Arsenic groundwater has far reaching consequences 
including its ingestion through food chain, which are in the form of social disorders, health hazards and socioeconomic dissolution besides its sprawling with movement and exploitation of groundwater. The food crops which are grown using arsenic contaminated water are sold off to other places, includ ing contaminated regions where the inhabitants may consume arsenic from contaminated food. This may give rise to new danger.

It is well known that trace elements of Arsenic is both advantageous to plant and animal nutrition[4,5,6], but there are no reports of this type in humans[7]. However, higher levels of Arsenic are found to be harmful to humans. The contamination of Arsenic of surface and groundwater occurs worldwide and has become a sociopolitical issue in several parts of the globe. For example, there are lots of people who are at risk of drinking As-contaminated water in West Bengal (India)[8,9] and Bangladesh[10]. Scores of people from China[11], Vietnam[12], Taiwan[13], Chile[14], Argentina[15], and Mexico[16] are likely at risk as well. Skin manifestations of many types and other arsenic toxicity were observed from melanosis, keratosis, hyperkeratosis, dorsal keratosis, and non pitting edema to gangrene and cancer. Overall, prevalence of clinical neuropathy was noticed in various studies in populations of 24Pargana-North, 24- Pargana-South, Murshidabad, Nadia, and Bardhaman districts of West Bengal and in the states of Bihar, Uttar Pradesh, Jharkhand and Chhattis garh. Adults are less affected due to arsenic than children. Most of the population suffering from arsenic skin lesions is from a poor socio-economic background and adults are less affected to arsenic than children. Albertus Magnus in 1250 AD for the first time documented the hazardous effects of Arsenic . The hazardous effects of Arsenic on both flora and fauna are well known[17]. The consumption of arsenic contaminated water is the main path for its transportation into the environment and biological systems $[3,18,19,20,21]$ is also well known. Attempts have been made to determine and establish a database on the drinking water quality of Shuklaganj area with particular emphasis on the physico-chemical characteristics and levels of heavy metals in the water samples.

\section{Materials and Methods}

Table 1. Shows GPS location and physico - chemical properties of water collected from handpumps around Shuklaganj area in premonsoon

\begin{tabular}{|c|c|c|c|c|c|c|c|c|c|c|}
\hline S.N. & GPS Location & Location & $\mathrm{pH}$ & $\begin{array}{c}\text { Temp } \\
{ }^{\circ} \mathrm{C}\end{array}$ & $\begin{array}{c}\text { Hardness } \\
\mathrm{mg} / 1\end{array}$ & $\begin{array}{c}\text { Alkalinity } \\
\mathrm{mg} / \mathrm{l}\end{array}$ & $\begin{array}{c}\text { Conduct ivity } \\
\mu \mathrm{s} / \mathrm{cm}\end{array}$ & $\begin{array}{l}\mathrm{TDS} \\
\mathrm{mg} / \mathrm{l}\end{array}$ & $\begin{array}{c}\text { Salinity } \\
\text { ppt }\end{array}$ & $\mathrm{Cl}-$ \\
\hline 1 & $\begin{array}{l}\text { N260 '29.925' } \\
\text { E0800 '27.594' }\end{array}$ & \multirow{4}{*}{$\begin{array}{l}\text { Gajiyakheda } \\
\text { (Shuklaganj) }\end{array}$} & 8.5 & 25 & 200 & 100 & 872 & 438 & 0.3 & 20.2 \\
\hline 2 & $\begin{array}{c}\text { N260 '29.925' } \\
\text { E0800 '27.594' }\end{array}$ & & 8.5 & 25 & 180 & 88 & 828 & 412 & 0.2 & 16.2 \\
\hline 3 & $\begin{array}{l}\text { N260 '28.616' } \\
\text { E0800 '23.046' }\end{array}$ & & 7.5 & 25 & 180 & 112 & 570 & 285 & 0.0 & 12.1 \\
\hline 4 & $\begin{array}{l}\text { N260 '28.066' } \\
\text { E0800 '22.246' }\end{array}$ & & 7.5 & 25 & 212 & 100 & 538 & 275 & 0.2 & 18.2 \\
\hline 5 & $\begin{array}{l}\text { N260 '28.069' } \\
\text { E0800 '22.248' }\end{array}$ & \multirow{5}{*}{$\begin{array}{c}\text { Majhara } \\
\text { pipalkheda } \\
\text { (Shuklaganj) }\end{array}$} & 7.5 & 25 & 180 & 84 & 466 & 229 & 0.2 & 16.2 \\
\hline 6 & $\begin{array}{l}\text { N260 '28.668' } \\
\text { E0800 '22.363' }\end{array}$ & & 7.5 & 25 & 200 & 92 & 587 & 293 & 0.1 & 32.3 \\
\hline 7 & $\begin{array}{c}\text { N260 '28.659' } \\
\text { E0800 '22.363' }\end{array}$ & & 8.5 & 25 & 200 & 84 & 320 & 164 & 0.0 & 18.2 \\
\hline 8 & $\begin{array}{l}\text { N260 '28.537' } \\
\text { E0800 '22.468' }\end{array}$ & & 8.5 & 25 & 212 & 100 & 322 & 163.6 & 0.0 & 14.1 \\
\hline 9 & $\begin{array}{l}\text { N260 '28.486', } \\
\text { E0800 '22.869' }\end{array}$ & & 8.5 & 25 & 208 & 96 & 676 & 337 & 0.2 & 14.1 \\
\hline 10 & $\begin{array}{l}\text { N260 '29.137' } \\
\text { E0800 '23.542' }\end{array}$ & $\begin{array}{l}\text { Jabbupurwa } \\
\text { (Shuklaganj) }\end{array}$ & 8.5 & 25 & 180 & 88 & 934 & 466 & 0.3 & 32.3 \\
\hline 11 & $\begin{array}{l}\text { N260 '29.389' } \\
\text { E0800 '23.633' }\end{array}$ & $\begin{array}{l}\text { Poni Bajar } \\
\text { (Shuklaganj) }\end{array}$ & 7.5 & 25 & 200 & 108 & 1028 & 514 & 0.4 & 12.1 \\
\hline 12 & $\begin{array}{l}\text { N260 '29.331', } \\
\text { E0800 '23.655' }\end{array}$ & $\begin{array}{c}\text { Poni(Primary } \\
\text { school, } \\
\text { Shuklaganj) }\end{array}$ & 7.5 & 25 & 208 & 108 & 1284 & 643 & 0.6 & 18.2 \\
\hline 13 & $\begin{array}{l}\text { N260 '29.352' } \\
\text { E0800 '23.698' }\end{array}$ & \multirow{5}{*}{$\begin{array}{l}\text { Nihalkheda } \\
\text { (Shuklaganj) }\end{array}$} & 7.5 & 25 & 200 & 100 & 2140 & 1102 & 0.3 & 16.2 \\
\hline 14 & $\begin{array}{l}\text { N260 '29.430' } \\
\text { E0800 '23.844' }\end{array}$ & & 7.5 & 25 & 180 & 112 & 1094 & 547 & 0.4 & 12.1 \\
\hline 15 & $\begin{array}{c}\text { N260 '29.391' } \\
\text { E0800 '20.824' }\end{array}$ & & 8.0 & 25 & 200 & 100 & 1242 & 619 & 0.6 & 32.3 \\
\hline 16 & $\begin{array}{l}\text { N260 '27.984' } \\
\text { E0800 '25.891' }\end{array}$ & & 7.5 & 25 & 212 & 96 & 902 & 452 & 0.3 & 18.2 \\
\hline 17 & $\begin{array}{c}\text { N260 '27.953' } \\
\text { E0800 '25.831' }\end{array}$ & & 7.5 & 25 & 200 & 92 & 1892 & 960 & 1.0 & 36.4 \\
\hline
\end{tabular}

The data is the mean of three samples collected from each source $(\mathrm{N}=3)$ 
Table 2. Shows GPS location and physico - chemical properties of water collected from handpumps around Shuklaganj area in the postmonsoon

\begin{tabular}{|c|c|c|c|c|c|c|c|c|c|c|}
\hline S.N & GPS Location & Location & $\mathrm{pH}$ & $\underset{{ }^{\circ} \mathrm{C}}{\text { Temp }}$ & $\begin{array}{l}\text { Hardness } \\
\mathrm{mg} / \mathrm{l}\end{array}$ & $\begin{array}{c}\text { Alkalinity } \\
\mathrm{mg} / \mathrm{l}\end{array}$ & $\begin{array}{c}\text { Conductivity } \\
\mu \mathrm{s} / \mathrm{cm}\end{array}$ & $\begin{array}{l}\text { TDS } \\
\mathrm{mg} / 1\end{array}$ & $\begin{array}{l}\text { Salinity } \\
\text { ppt }\end{array}$ & $\mathrm{Cl}^{-}$ \\
\hline 1 & $\begin{array}{l}\text { N26 } 6^{\circ} 29.025, \\
\text { E080 } \\
\end{array}$ & $\begin{array}{l}\text { Maheshkheda } \\
\text { (Shuklaganj) }\end{array}$ & 7.3 & 26.8 & 210 & 72 & 1944 & 1019 & 1.3 & 38.4 \\
\hline 2 & $\begin{array}{l}\mathrm{N} 26^{0} 29.605^{\prime} \\
\mathrm{E} 080^{\circ} 24.240^{\prime}\end{array}$ & \multirow{2}{*}{$\begin{array}{l}\text { Nayakheda } \\
\text { (Shuklaganj) }\end{array}$} & 7.5 & 27.0 & 190 & 76 & 633 & 317 & 0.3 & 16.2 \\
\hline 3 & $\begin{array}{l}\text { N26 }{ }^{\circ} \cdot 29.357 \\
\text { E080 }\end{array}$ & & 7.3 & 28.2 & 170 & 96 & 861 & 430 & 0.3 & 40.4 \\
\hline 4 & $\begin{array}{l}\text { N26 } 29.039^{\prime} \\
\text { E080 } \\
0^{\circ} 23.750^{\prime}\end{array}$ & $\begin{array}{l}\text { Panchwati } \\
\text { Mandir } \\
\text { (Shuklaganj) }\end{array}$ & 7.3 & 28.1 & 150 & 76 & 432 & 230 & 0.0 & 10.1 \\
\hline 5 & $\begin{array}{l}\text { N26 } 28.580^{\prime} \\
\text { E080 } 22.994^{\prime}\end{array}$ & $\begin{array}{l}\text { Swaraswati } \\
\text { palace } \\
\text { (Shuklaganj) }\end{array}$ & 7.4 & 28.4 & 180 & 116 & 1281 & 639 & 0.8 & 38.4 \\
\hline 6 & $\begin{array}{l}\text { N26 }{ }^{0} 29.191^{\prime} \\
\text { E}^{\prime} 80^{\circ} 23.601^{\prime}\end{array}$ & \multirow{4}{*}{ Shuklaganj } & 7.4 & 28.1 & 170 & 76 & 488 & 244 & 0.2 & 10.1 \\
\hline 7 & $\begin{array}{l}\text { N26 '29.159' } \\
\text { E080 } 23.560\end{array}$ & & 7.3 & 28.2 & 190 & 116 & 467 & 233 & 0.2 & 16.2 \\
\hline 8 & $\begin{array}{l}\text { N26 }{ }^{\circ} 29.657^{\prime} \\
\text { E080 } 24.058^{\circ}\end{array}$ & & 7.4 & 28.4 & 210 & 116 & 898 & 427 & 0.5 & 404 \\
\hline 9 & $\begin{array}{c}\mathrm{N} 26^{0} 28.485 \\
\mathrm{E} 080^{0} 22.836^{\prime}\end{array}$ & & 7.8 & 27.5 & 130 & 112 & 1672 & 839 & 1.1 & 36.4 \\
\hline 10 & $\begin{array}{l}\mathrm{N} 26^{0} \\
\text { E080 } 28.699 \\
\text { E } 22.382^{\prime}\end{array}$ & $\begin{array}{l}\text { Mishra colony } \\
\text { (Swarg dham) }\end{array}$ & 7.3 & 27.0 & 170 & 84 & 847 & 422 & 05 & 38.4 \\
\hline 11 & $\begin{array}{l}\mathrm{N} 26^{0} 28.632^{\prime} \\
\mathrm{E} 080^{\circ} 22.518^{\prime}\end{array}$ & $\begin{array}{l}\text { Mishra colony } \\
\text { (Ganga ghat) }\end{array}$ & 7.0 & 27.4 & 190 & 88 & 1558 & 786 & 1.0 & 32.3 \\
\hline 12 & $\begin{array}{l}\mathrm{N} 26^{0} 29.096^{\prime} \\
\mathrm{E} 080^{0} 23.081\end{array}$ & Champapurwa & 7.4 & 28.3 & 180 & 76 & 559 & 279 & 0.2 & 20.2 \\
\hline 13 & $\begin{array}{l}\text { N26 } 6^{0} 28.946^{\prime} \\
\text { E080 } \\
\end{array}$ & $\begin{array}{l}\text { Champapurwa } \\
\text { (manshukheda) }\end{array}$ & 7.1 & 27.3 & 140 & 108 & 1055 & 526 & 0.6 & 34.5 \\
\hline
\end{tabular}

The data is the mean of three samples collected from each source $(\mathrm{N}=3)$

Drinking water samples were collected from India mark II handpumps in and around Shuklaganj area. Samples for physico-chemical analysis were collected in plastic sterilized bottles and transported to the laboratory. Before filling the samples these bottles have been rinsed two or three times with water. The record of every sample is maintained by an appropriate labelling including the name of the sample collector, the date, timing and exact location. Identification of the sites was made by recording the co-ordinates using the GPS. The samples were collected in premonsoon and postmonsoon of Shuklaganj area. For analysis of metals the water samples have been collected in glass or plastic (polyethylene) bottles and $2.0 \mathrm{ml}$ of nitric acid is added in each bottle. All the samples have to be stored at $4^{0} \mathrm{C}$ for storage and analysis. For quantitative metal analysis a multi-elemental standard solution of $\mathrm{Cu}, \mathrm{Cr}, \mathrm{Cd}, \mathrm{Zn}, \mathrm{Fe}, \mathrm{Mn}$, $\mathrm{Ni}$, As was collected in $2 \%$ nitric acid commercial $1 \mathrm{~g} / \mathrm{L}$. Individual standard solution was stored in polyethylene bottles. Measurement was made on Inductivity Couple Plas ma (ICP) Instrument (Thermo Electric Corporation 
Intrepid II $x$ DL) with axial viewing configuration. The complete process of sample preparation and analysis of physico-chemical and metals was made as per the standard methods[22].

\section{Results and Discussion}

The physico-chemical parameters and the GPS location of the water samples collected in and around Shuklaganj area are shown in Table1,2,3. The parameters determined were $\mathrm{pH}$, hardness, alkalinity, conductivity, TDS, salinity and chloride content. The samples were collected in premonsoon and postmonsoon of Shuklaganj area. The $\mathrm{pH}$ of all the samples varied from 7.0 to 8.5 in both premonsoon and postmonsoon period. Hardness during premonsoon varied from $180-212 \mathrm{mg} / \mathrm{L}$ and in postmonsoon varied from $140-210 \mathrm{mg} / \mathrm{l}$. A kalinity varied from $84-112 \mathrm{mg} / \mathrm{L}$ in premonsoon and $60-128 \mathrm{mg} / \mathrm{L}$ in postmosoon. However conductivity was quite high and it ranged from 320-2140 $\mu \mathrm{s} / \mathrm{cm}$ in premonsoon and $358-1944 \mu \mathrm{s} / \mathrm{cm}$ during postmonsoon. The TDS ranged from 181-1019 $\mathrm{mg} / 1$ in premonsoon samples and $163-1102 \mathrm{mg} / 1$ in postmonsoon. Salinity varied from $0.0-1.0 \mathrm{ppt}$ in premonsoon and $0.0-1.3$ ppt in postmonsoon. Chloride content varied from to 12.1-36.4 in premonsoon and $8.08-44.5 \mathrm{mg} / \mathrm{L}$ in potmonsoon.

Table 3. Shows GPS location and physico - chemical properties of water collected from handpumps around Shuklaganj area in postmonsoon

\begin{tabular}{|c|c|c|c|c|c|c|c|c|c|c|}
\hline $\begin{array}{l}\mathrm{S} . \\
\mathrm{N}\end{array}$ & GPS Location & Location & $\mathrm{pH}$ & $\begin{array}{c}\text { Temp } \\
{ }^{\circ} \mathrm{C}\end{array}$ & $\begin{array}{c}\text { Hardness } \\
\mathrm{mg} / \mathrm{l}\end{array}$ & $\begin{array}{c}\text { Alkalinity } \\
\mathrm{mg} / \mathrm{l}\end{array}$ & $\begin{array}{c}\text { Conduct ivity } \\
\mu \mathrm{s} / \mathrm{cm}\end{array}$ & $\begin{array}{l}\text { TDS } \\
\mathrm{mg} / 1\end{array}$ & $\begin{array}{c}\text { Salinity } \\
\text { ppt }\end{array}$ & $\mathrm{Cl}^{-}$ \\
\hline 1 & $\begin{array}{l}\text { N26 }{ }^{\circ} 29.925^{\circ} \\
\mathrm{E} 080^{\circ} 27.594,\end{array}$ & $\begin{array}{l}\text { Ambikapuram } \\
\text { (Shuklaganj) }\end{array}$ & 7.5 & 25 & 180 & 128 & 1086 & 539 & 0.6 & 16.2 \\
\hline 2 & $\begin{array}{l}\text { N26 }{ }^{0} 29.925^{\prime} \\
\text { E080 }\end{array}$ & $\begin{array}{l}\text { Baunamau } \\
\text { (Shuklaganj) }\end{array}$ & 7.5 & 25 & 130 & 76 & 538 & 269 & 0.1 & 26.3 \\
\hline 3 & $\begin{array}{l}\text { N26 }{ }^{\circ} 28.616^{\prime} \\
\text { E080 } \\
\end{array}$ & $\begin{array}{l}\text { Shaheen Market } \\
\text { (Shuklaganj) }\end{array}$ & 8.5 & 25 & 210 & 80 & 712 & 356 & 0.2 & 18.2 \\
\hline 4 & $\begin{array}{l}\text { N26 }{ }^{\circ} 28.066^{\prime} \\
\text { E080 } \\
\end{array}$ & $\begin{array}{c}\text { Ganga Pul } \\
\text { (Shuklaganj) }\end{array}$ & 7.5 & 26 & 140 & 36 & 1139 & 567 & 0.5 & 24.3 \\
\hline 5 & 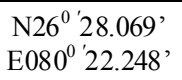 & $\begin{array}{l}\text { Mishra colony } \\
\text { (Entrance) }\end{array}$ & 7.5 & 26 & 190 & 76 & 1202 & 600 & 0.6 & 22.2 \\
\hline 6 & $\begin{array}{l}\text { N26 } 6^{\circ} 28.668^{\prime}, \\
\text { E080 }\end{array}$ & $\begin{array}{l}\text { Mishra colony } \\
\text { (Ghat ke pass) }\end{array}$ & 8.5 & 25 & 170 & 120 & 754 & 376 & 0.2 & 32.3 \\
\hline 7 & $\begin{array}{l}\text { N26 '28.659', } \\
\text { E080 } 22.363,\end{array}$ & $\begin{array}{l}\text { Mishra colony } \\
\text { (Ganga ghat) }\end{array}$ & 8.0 & 25 & 170 & 128 & 1361 & 678 & 0.6 & 44.5 \\
\hline 8 & $\begin{array}{l}\text { N26 }{ }^{\circ} 28.537^{\prime}, \\
\text { E080 } 22.468\end{array}$ & $\begin{array}{c}\text { Mishra colony } \\
\text { (Naveen Badh } \\
\text { kendra) } \\
\end{array}$ & 7.5 & 25 & 180 & 128 & 1340 & 670 & 0.6 & 44.5 \\
\hline 9 & 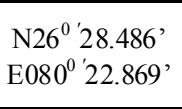 & $\begin{array}{c}\text { Shuklaganj } \\
\text { (Kafibar,Thane ke } \\
\text { pass) }\end{array}$ & 7.5 & 25 & 130 & 100 & 1068 & 533 & 0.5 & 38.4 \\
\hline 10 & $\begin{array}{l}\text { N26 '29.137', } \\
\text { E080 } 23.542 \text { ' }\end{array}$ & $\begin{array}{c}\text { Shuklaganj } \\
\text { (rajdhani Road } \\
\text { Mandir) } \\
\end{array}$ & 7.5 & 25 & 210 & 92 & 388 & 194.1 & 0.1 & 10.1 \\
\hline 11 & $\begin{array}{l}\text { N26 } 29.389^{\circ}, \\
\text { E080 } 23.633,\end{array}$ & $\begin{array}{c}\text { Shuklaganj } \\
\text { (Primary } \\
\text { School,Netuwa) } \\
\end{array}$ & 7.0 & 26 & 150 & 60 & 422 & 211 & 0.1 & 20.2 \\
\hline 12 & $\begin{array}{l}\text { N26 '29.331', } \\
\text { E080' } 23.655 \text { ' }\end{array}$ & $\begin{array}{c}\text { Shuklaganj } \\
\text { (Panchayat } \\
\text { Bhawan Netuwa) }\end{array}$ & 8.5 & 26 & 170 & 48 & 358 & 181.6 & 0.1 & 8.08 \\
\hline 13 & $\begin{array}{l}\text { N26 } 29.352 \text { ' } \\
\text { E080 } 23.698,\end{array}$ & $\begin{array}{c}\text { Shuklaganj } \\
\text { (Rashmilok } \\
\text { Netuwa) } \\
\end{array}$ & 8.0 & 26 & 190 & 124 & 616 & 314 & 0.1 & 22.2 \\
\hline 14 & $\begin{array}{l}\text { N26 } 0^{\circ} 29.430^{\prime}, \\
\text { E080 }\end{array}$ & $\begin{array}{l}\text { Shuklaganj } \\
\text { (Sarosi) }\end{array}$ & 8.0 & 26 & 170 & 120 & 796 & 398 & 0.3 & 24.5 \\
\hline 15 & $\begin{array}{l}\text { N26 } 29.391 \text { ' } \\
\text { E080 } 20.824 \text { ' }\end{array}$ & $\begin{array}{c}\text { Shuklaganj } \\
\text { (Sarosi, Sulabh } \\
\text { Sauchalay) } \\
\end{array}$ & 7.5 & 25 & 170 & 72 & 495 & 248 & 0.1 & 28.3 \\
\hline 16 & $\begin{array}{l}\mathrm{N}^{2} 6^{0} \text { '27.984', } \\
\text { E080 } 25.891\end{array}$ & \multirow{2}{*}{$\begin{array}{l}\text { Poni Bajaar, } \\
\text { Bypaas road }\end{array}$} & 7.5 & 25 & 130 & 124 & 511 & 255 & 0.1 & 38.4 \\
\hline 17 & $\begin{array}{l}\text { N26 } 6^{\circ} 27.953^{\prime}, \\
\text { E080 } 0^{\circ} 25.831,\end{array}$ & & 7.5 & 25 & 150 & 60 & 711 & 355 & 0.2 & 30.3 \\
\hline
\end{tabular}

The data is the mean of three samples collected from each source $(\mathrm{N}=3)$ 
Table 4. Shows the heavy metals and arsenic present in water collected from handpumps in and around Shuklaganj in premonsoon

\begin{tabular}{|c|c|c|c|c|c|c|c|c|c|}
\hline S.N & Location & $\mathrm{Cu}$ & $\mathrm{Cr}$ & $\mathrm{Cd}$ & $\mathrm{Zn}$ & $\mathrm{Fe}$ & $\mathrm{Mn}$ & $\mathrm{Ni}$ & As \\
\hline 1 & \multirow{4}{*}{$\begin{array}{l}\text { Gajiyakheda } \\
\text { (Shuklaganj) }\end{array}$} & $\mathrm{BDL}$ & BDL & 0.0007 & BDL & BDL & BDL & BDL & 10 \\
\hline 2 & & $\mathrm{BDL}$ & BDL & $\mathrm{BDL}$ & BDL & BDL & $\mathrm{BDL}$ & 0.0018 & 25 \\
\hline 3 & & 0.0178 & 0.0083 & BDL & 2.46 & 16.61 & 0.1711 & BDL & 25 \\
\hline 4 & & 0.0057 & BDL & BDL & 2.86 & 10.56 & 0.0854 & BDL & 250 \\
\hline 5 & \multirow{5}{*}{$\begin{array}{l}\text { Majharapipalkheda } \\
\text { (Shuklaganj) }\end{array}$} & 0.0057 & BDL & BDL & 2.35 & 12.30 & 0.2154 & 0.0009 & 5 \\
\hline 6 & & 0.0009 & BDL & BDL & BDL & 16.23 & 0.2791 & BDL & 0 \\
\hline 7 & & 0.0107 & BDL & BDL & 3.41 & 4.34 & 0.235 & BDL & 10 \\
\hline 8 & & 0.0137 & BDL & BDL & 3.36 & 5.50 & 0.2117 & BDL & 25 \\
\hline 9 & & 0.0005 & BDL & BDL & 1.15 & 6.56 & 0.1472 & BDL & 0 \\
\hline 10 & $\begin{array}{l}\text { Jabbupurwa } \\
\text { (Shuklaganj) }\end{array}$ & 0.0005 & BDL & BDL & 1.23 & 7.41 & 0.1208 & BDL & 0 \\
\hline 11 & $\begin{array}{l}\text { Poni Bajar } \\
\text { (Shuklaganj) }\end{array}$ & 0.0012 & BDL & BDL & 0.0481 & 5.97 & 0.0945 & BDL & 50 \\
\hline 12 & $\begin{array}{c}\text { Poni(Primary } \\
\text { school, Shuklaganj) }\end{array}$ & 0.001 & BDL & BDL & BDL & 3.16 & 0.1999 & 0.0014 & 0 \\
\hline 13 & \multirow{5}{*}{$\begin{array}{l}\text { Nihalkheda } \\
\text { (Shuklaganj) }\end{array}$} & 0.0142 & BDL & BDL & 3.26 & 8.93 & 0.4363 & BDL & 10 \\
\hline 14 & & 0.0048 & $\mathrm{BDL}$ & BDL & 2.77 & 13.28 & 0.1957 & BDL & 25 \\
\hline 15 & & BDL & BDL & BDL & BDL & 8.45 & 0.1585 & BDL & 50 \\
\hline 16 & & 0.0078 & 0.0062 & BDL & 3.09 & 17.82 & 0.3709 & BDL & 250 \\
\hline 17 & & 0.0107 & 0.0012 & BDL & 2.24 & 17.99 & 0.4454 & BDL & 100 \\
\hline
\end{tabular}

The data is the mean of three samples collected from each source $(\mathrm{N}=3)$

The picture of heavy metals and arsenic present in the water collected during premonsoon and postmonsoon periods were also determined and are shown in Table 4,5,6. However, in premonsoon samples $\mathrm{Cr}, \mathrm{Cd}$ and $\mathrm{Ni}$ were absent in most of the samples. It was noticed that $\mathrm{Cd}$ and $\mathrm{Ni}$ content was almost absent in the samples collected during postmonsoon season. Copper varied from 0.0 to 0.0178 $\mathrm{mg} / \mathrm{L}$ during premonsoon and 0.0002 to $0.0098 \mathrm{mg} / \mathrm{L}$ during postmonsoon. Zinc content varied from 0.0 to 3.26 in premonsoon period and from $0.0-3.6 \mathrm{mg} / \mathrm{L}$ in postmonsoon period. Iron varied from 0.0 to $17.99 \mathrm{mg} / \mathrm{L}$ in pre monsoon and varied from 0.0692 to 12.53 in postmonsoon samples. Manganese varied from 0.0 to $0.4454 \mathrm{mg} / \mathrm{L}$ in premonsoon samples and $0.0018-4.74 \mathrm{mg} / \mathrm{L}$ in premonsoon samples. Arsenic in premonsoon seas on varied from $0-250 \mathrm{ppb}$ and from $0-250 \mathrm{ppb}$ in postmonsoon.

Arsenic is stable in four oxidation states $(+5,+3,0,-3)$ under the Eh conditions that occur in aquatic systems. At high Eh values (mostly exist in oxygenated waters), arsenic acid species (i.e., $\mathrm{H}_{3} \mathrm{AsO}_{4}, \mathrm{H}_{2} \mathrm{AsO}_{4}{ }^{-}, \mathrm{HAsO}_{4}{ }^{2-}$, and $\mathrm{AsO}_{4}{ }^{3-}$ ) are stable. At mildly reducing conditions, arsenious acid species (i.e., $\mathrm{H}_{3} \mathrm{AsO}_{3}, \mathrm{H}_{2} \mathrm{AsO}_{3}^{-}$, and $\mathrm{HAsO}_{3}^{-2}$ ) become 
stable[23,24,25]. The speciation of As in aquatic environment is critical in controlling the adsorption/desorpti on reactions with sediments. Adsorption to sediment particles may remove $\mathrm{As}(\mathrm{V})$ from contaminated water, as well as inhibiting the precipitation of As minerals such as scorodite $\left(\mathrm{FeAsO}_{4} . \mathrm{H}_{2} \mathrm{O}\right)$ that control the equilibrium aqueous concentration[26]. Under the aerobic and acidic to near-neutral conditions (typical of many aquatic environments), As(V) is adsorbed very strongly by oxide minerals in sediments. The highly nonlinear nature of the adsorption isotherm for $\mathrm{As}(\mathrm{V})$ in oxide minerals ensures that the amount of As adsorbed is relatively large, even when dissolved aqueous concentrations of As are low. Such adsorption occurring in natural environments protects water bodies from widespread As toxic ity problems. Adsorption of As species by sediments are as follows: As(V) $>$ As(III) $>$ As
(II) $>$ DMA[3]. In As-contaminated sediments, Clement and Faust (1981)[27] found that a significant portion of the As was bound in organo-complex forms and indicated that adsorption-desorption equilibrium must be considered as well as the redox effects in examining the dynamics of As in aquatic environment.

\section{Conclusions}

It is seen that with increase of $\mathrm{pH}$ above 8.5, Arsenic desorbs from the oxide surfaces, thereby increasing the concentration of As in solution. It is suggested that the most desirable and significant mechanism for the groundwater As problems due to oxidizing conditions and the desorption of As from As contaminated sediments at high $\mathrm{pH}[28,29]$.

Table 5. Shows the heavy metals and arsenic present in water collected from handpumps around Shuklaganj in postmonsoon

\begin{tabular}{|c|c|c|c|c|c|c|c|c|c|}
\hline S.N. & Location & $\mathrm{Cu}$ & $\mathrm{Cr}$ & $\mathrm{Cd}$ & $\mathrm{Zn}$ & $\mathrm{Fe}$ & Mn & $\mathrm{Ni}$ & $\begin{array}{l}\text { As } \\
(\mathrm{PPb})\end{array}$ \\
\hline 1. & $\begin{array}{l}\text { Maheshkheda } \\
\text { (Shuklaganj) }\end{array}$ & 0.002 & BDL & 0.0004 & 0.2823 & 8.20 & 0.4695 & 0.0012 & 5 \\
\hline 2. & & 0.0029 & BDL & BDL & 0.059 & 12.53 & 0.0618 & BDL & 0 \\
\hline 3. & & 0.0017 & $\mathrm{BDL}$ & BDL & 0.032 & 0.2378 & 0.1104 & 0.0009 & 5 \\
\hline 4. & $\begin{array}{l}\text { Panchwati Mandir } \\
\text { (Shuklaganj) }\end{array}$ & BDL & BDL & BDL & $\mathrm{BDL}$ & 0.0692 & 0.0598 & BDL & 0 \\
\hline 5. & $\begin{array}{l}\text { Swaraswati palace } \\
\text { (Shuklaganj) }\end{array}$ & 0.0037 & BDL & 0.0008 & 0.2506 & 6.88 & 0.025 & 0.0087 & 0 \\
\hline 6. & \multirow{4}{*}{ Shuklaganj } & 0.0002 & BDL & $\mathrm{BDL}$ & 0.2709 & BDL & 0.4954 & BDL & 250 \\
\hline 7. & & 0.003 & BDL & 0.0001 & 0.0564 & 0.2795 & 0.2052 & 0.0027 & 0 \\
\hline 8. & & 0.0026 & 0.0053 & 0.0009 & 0.0242 & 0.2655 & 0.0955 & 0.0012 & 0 \\
\hline 9. & & 0.0015 & $\mathrm{BDL}$ & $\mathrm{BDL}$ & $\mathrm{BDL}$ & BDL & 0.0034 & 0.0005 & 250 \\
\hline 10. & $\begin{array}{c}\text { Mishra colony (Swarg } \\
\text { dham) }\end{array}$ & 0.002 & 0.0107 & 0.0001 & 0.0261 & 0.0206 & 0.3665 & 0.0003 & 250 \\
\hline 11. & $\begin{array}{l}\text { Mishra colony (Ganga } \\
\text { ghat) }\end{array}$ & 0.005 & BDL & BDL & $\mathrm{BDL}$ & BDL & 0.0018 & $\mathrm{BDL}$ & 250 \\
\hline 12. & Champapurwa & 0.0066 & BDL & 0.0004 & 3.60 & 5.24 & 4.74 & 0.003 & 25 \\
\hline 13. & $\begin{array}{l}\text { Champapurwa } \\
\text { (manshukheda) }\end{array}$ & 0.0018 & 0.0056 & BDL & 0.0035 & 0.0766 & 0.3665 & BDL & 0 \\
\hline
\end{tabular}

The data is the mean of three samples collected from each source $(\mathrm{N}=3)$ 
Table 6. Shows the heavy metals and arsenic present in water collected from handpumps in and around Shuklaganj in postmonsoon

\begin{tabular}{|c|c|c|c|c|c|c|c|c|c|}
\hline S.N & Location & $\mathrm{Cu}$ & $\mathrm{Cr}$ & $\mathrm{Cd}$ & $\mathrm{Zn}$ & $\mathrm{Fe}$ & $\mathrm{Mn}$ & $\mathrm{Ni}$ & As \\
\hline 1. & $\begin{array}{l}\text { Ambikapuram } \\
\text { (Shuklaganj) }\end{array}$ & 0.0098 & 0.095 & BDL & 6.26 & 0.2213 & 4.53 & 0.0138 & 0 \\
\hline 2. & $\begin{array}{l}\text { Baunamau } \\
\text { (Shuklaganj) }\end{array}$ & 0.0039 & 0.0363 & BDL & 5.44 & 19.3 & 0.2967 & BDL & 250 \\
\hline 3. & $\begin{array}{l}\text { Shaheen Market } \\
\text { (Shuklaganj) }\end{array}$ & 0.0017 & 0.0149 & 0.0015 & 5.87 & 21.33 & 0.0215 & 0.0073 & 0 \\
\hline 4. & $\begin{array}{l}\text { Ganga Pul } \\
\text { (Shuklaganj) }\end{array}$ & BDL & 0.0164 & BDL & 0.0247 & 3.25 & 0.0246 & $\mathrm{BDL}$ & 250 \\
\hline 5. & $\begin{array}{l}\text { Mishra colony } \\
\text { (Entrance) }\end{array}$ & BDL & 0.0169 & BDL & 0.0485 & 15.19 & 0.2328 & BDL & 25 \\
\hline 6. & $\begin{array}{c}\text { Mishra colony (Ghat ke } \\
\text { pass) }\end{array}$ & 0.0079 & 0.0135 & BDL & BDL & 0.0194 & 0.001 & $\mathrm{BDL}$ & 0 \\
\hline 7. & $\begin{array}{l}\text { Mishra colony (Ganga } \\
\text { ghat) }\end{array}$ & 0.0005 & 0.0071 & $\mathrm{BDL}$ & BDL & 0.000 & 0.000 & $\mathrm{BDL}$ & 0 \\
\hline 8. & $\begin{array}{l}\text { Mishra colony (Naveen } \\
\text { Badh kendra) }\end{array}$ & BDL & 0.0282 & 0.0001 & 3.82 & 11.27 & 0.0185 & $\mathrm{BDL}$ & 50 \\
\hline 9. & $\begin{array}{c}\text { Shuklaganj } \\
\text { (Kafibar,Thane ke } \\
\text { pass) }\end{array}$ & 0.0026 & 0.0329 & BDL & 0.0849 & 7.36 & 0.0295 & $\mathrm{BDL}$ & 0 \\
\hline 10. & $\begin{array}{l}\text { Shuklaganj (rajdhani } \\
\text { Road Mandir) }\end{array}$ & BDL & 0.0051 & BDL & 5.09 & 0.0161 & 0.1002 & BDL & 50 \\
\hline 11. & $\begin{array}{l}\text { Shuklaganj Primary } \\
\text { School,Netuwa) }\end{array}$ & 0.0007 & 0.0277 & BDL & 4.99 & 10.26 & 0.1041 & $\mathrm{BDL}$ & 250 \\
\hline 12. & $\begin{array}{c}\text { Shuklaganj (Panchayat } \\
\text { Bhawan, Netuwa) }\end{array}$ & 0.0012 & 0.0201 & BDL & 4.91 & 21.45 & 0.1052 & BDL & 25 \\
\hline 13. & $\begin{array}{c}\text { Shuklaganj (Rashmilok } \\
\text { Netuwa) }\end{array}$ & 0.0003 & 0.0218 & BDL & 5.81 & 11.52 & 0.1370 & BDL & 250 \\
\hline 14. & Shuklaganj (Sarosi, ) & 0.0003 & 0.033 & $\mathrm{BDL}$ & 4.58 & 19.86 & 0.1726 & $\mathrm{BDL}$ & 250 \\
\hline 15. & $\begin{array}{l}\text { Shuklaganj (Sarosi, } \\
\text { Sulabh Sauchalay) }\end{array}$ & 0.0052 & 0.0255 & 0.0001 & 4.54 & 21.41 & 0.1984 & $\mathrm{BDL}$ & 50 \\
\hline 16. & \multirow{2}{*}{$\begin{array}{c}\text { Poni Bajaar, Bypaas } \\
\text { road }\end{array}$} & BDL & 0.0204 & BDL & 3.63 & 17.89 & 0.2137 & BDL & 25 \\
\hline 17. & & 0.0019 & 0.0019 & BDL & 0.0652 & 22.49 & 0.2636 & $\mathrm{BDL}$ & 100 \\
\hline
\end{tabular}

The data is the mean of three samples collected from each source $(\mathrm{N}=3)$

\section{ACKNOWLEDGEMENTS}

Thanks are due to UPCST, Lucknow for providing financial assistance.

\section{REFERENCES}

[1] USEPA, National primary drinking water regulations, arsenic and clarifications to compliance and new source contaminants monitoring final rule. Fed. Regis. 66 6976, 2001.
[2] World Health Organising. Guidelines for drinking water Quality. In Recommendations, vol 1, WHO, Geneva, 1993.

[3] P.L.Smedley, D.G. Kinniburgh, A review of the source, behavior and distribution of arsenic in natural waters. Appl. Geochem. 17, 517-568, 2002.

[4] Leonard A.. Arsenic. In "Metals and Their Compounds in the Environments: Occurrence, Analysis, and Biological Relevance (E. Merian, Ed.), 2nd edn, pp. 751-773, 1991. Weinheim, VCH.

[5] A. H.Smith, M. Goycolea, R. Haque, M.L.Biggs, Marked increase in bladder and lung cancer mortality in a region of 
northern Chile due to arsenic in drinking water.Am. J. Epidemiol. 147, 660-669, 1998.

[6] USEPA, United States Environmental Protection Agency, Integrated Risk InformationSystem on Arsenic. Office Health Environ Assess, Cincinnati, OH.Adriano, D. C., Page, A. L., Elseewi, A. A., Chang, A. C., and Straughan, I. (1980). Utilization and disposal of fly ash and other residues in terrestrial ecosystems: A review. J. Environ.Qual. 9, 333-344, 1993.

[7] Adriano D..C..,"Trace Elements in Terrestrial Environments Biogeochemistry, Bioavailability and Risks of Metals", 2nd edn, Springer, New York, 2001.

[8] D.Chakraborti, M.M. Rahman, K. Paul, U. K., Chowdhury, M.K. Sengupta, D. Lodh, C.R.Chanda, K.C., Saha, S.C. Mukherjee, Arsenic calamity in the Indian subcontinent: What lessons have been learned? Talanta 58, 3-22, 2002.

[9] A. Chatterjee, D.Das, B.K. Mandal, T.R. Chowdhury, G. Samanta, D.Chakraborti, Arsenic in ground-water in 6 districts of West-Bengal, India - the biggest arsenic calamity in the world. 1. Arsenic species in drinking-water and urine of the affected people. Analyst 120, 643-650,1995.

[10] A.H.Smith, O.E. Lingas, M. Rahman, Contamination of drinking water by arsenic in Bangladesh: A public health emergen cy. Bull. World Health Organ. 78, 1093-1103, 2000.

[11] G.Wang, Arsenic poisoning from drinking water in Xinjiang. Chin. J. Prevent. Med. 18, 105-107, 1984.

[12] M.Berg, H.C. Tran, T.C. Nguyen, H.V. Pham, R.Schertenleib, W. Giger, Arsenic contamination of groundwater and drinking water in Vietnam: A human health threat. Environ. Sci. Technol. 35, 2621-2626, 2001.

[13] F.J.Lu, Blackfoot disease: Arsenic or humic acid? Lancet 336, 115-119,1990.

[14] A.H.Smith, M.Goycolea, R. Haque, M.L. Biggs, Marked increase in bladder and lung cancer mortality in a region of northern Chile due to arsenic in drinking water. Am. J. Epidemiol. 147, 660-669, 1998.

[15] R.C.Hopenhayn, M.L.Biggs, D.A.Kalman, L.E.Moore, A.H.Smith, Arsenic methylation patterns before and after changing from high to lower concentrations of arsenic in drinking water. Environ. Health Perspect. 104, 1200-1207, 1996.

[16] L.M.Del Razo, M.A. Rellano, M.E. Cebrian, The oxidation states of arsenic in well-water from a chronic arsenicism area of northern M exico. Environ. Pollut. 64, 143-153, 1990.
[17] M.A. Khan, Y.S.Ho, Arsenic in drinking water : A review on toxicological effects, mechanism of accumulation and remediation. As. J.Chem.23,1889-1901, 2011.

[18] K.D. Huysmans, W.T.Jr Frankenberger, Evolution of trimethy larsine by a Penicillium sp. Isolated from agricultural evaporation pond water. Sci. Total Environ. 105, 13-28, 1991.

[19] M.Styblo, L.M.Del Razo, L.Vega, D.R.Germolec, E.L.Le Cluyse, G.A.Hamilton,W.Reed, C.Wang, W.R. Cullen and D.J. Thomas, Comparative toxicity of trivalent and pentavalent inorganic and methylated arsenicals in rat and human cells.Arch. Toxicol. 74, 289, 2000.

[20] F.W. Pointus, K.G.Brown, C.J.Chen, Adsorption of arsenic onto hydrous ferric oxide: effects of adsorbate/adsorbent ratios and co-occurring solutes.J.Am Water Work Assoc. 86, $52,1994$.

[21] National Research Council Uptake : National Academy Press : Washington DC 2001. http://www.nap.edu/books/030 $9076293 / \mathrm{html}$.

[22] APHA, Standard method for the examination of water and wastewater $21^{\text {st }}$ edition. American Public Health Asso. Washington DC, 2005.

[23] N.E.Korte, Q.Fernando, A review of arsenic (III) in groundwater. Crit. Rev.Environ. Control. 21, 1-39, 1991.

[24] W.R.Penrose, Arsenic in the marine and aquatic environments: Analysis, occurrence and significance. CRC Crit. Rev. Environ. Control 4, 465-482, 1974.

[25] D.G.Smith, Heavy metals in the New Zealand aquatic environment: A review. National Water and Soil Conservation Authority, Wellington, New Zealand. Water and Soil Miscellaneous Publication No. 100, 1986.

[26] A.L.Foster, G.E. Brown, G. A Parks, T.N. Tingle, D.E. Voigt, S.L. Brantley, XAFS determination of As(V) associated with $\mathrm{Fe}(\mathrm{III})$ oxyhydroxides in weathered mine tailings and contaminated soil from California, USA. J. De Phy sique IV 7, 815-816, 1997.

[27] W.H.Clement, S.D.Faust, The release of arsenic from contaminate sediments and muds. J. Environ. Sci. Health 1, 87-91, 1981.

[28] F.N.Robertson, Arsenic in groundwater under oxidizing conditions, southwest United States. Environ. Geochem. Health 11, 171-185, 1989.

[29] P.L.Smedley, H.B.Nicolli, D.M.J. Macdonald, A.J. Barros, J.O.Tullio, Hy drogeochemistry of arsenic and other inorganic constituents in groundwaters from La Pampa, Argentina. Appl. Geochem. 17, 259-284, 2002. 